\title{
Spontaneous Resolution of Chronic Subdural Hematoma : Close Observation as a Treatment Strategy
}

\author{
Hyung Chan Kim, M.D., ${ }^{1}$ Jung Ho Ko, M.D., Ph.D., ${ }^{1}$ Dong Soo Yoo, M.D., Ph.D., ${ }^{2}$ Sang-Koo Lee, M.D., Ph.D. \\ Departments of Neurological Surgery, ${ }^{1}$ Radiology, ${ }^{2}$ College of Medicine, Dankook University, Cheonan, Korea
}

\begin{abstract}
Objective : Chronic subdural hematoma (cSDH) is common condition in neurosurgical field. It is difficult to select the treatment modality between the surgical method and the conservative method when patients have no or mild symptoms. The purpose of this study is to provide a suggestion that the patients could be cured with conservative treatment modality.

Methods : We enrolled 16 patients who had received conservative treatment for cSDH without special medications which could affect hematoma resolution such as mannitol, steroids, tranexamic acid and angiotensin converting enzyme inhibitors. The patients were classified according to the Markwalder's Grading Scale.

Results : Among these 16 patients, 13 (81.3\%) patients showed spontaneously resolved cSDH and 3 (18.7\%) patients received surgery due to symptom aggravation and growing hematoma. They were categorized into two groups based on whether they were cured with conservative treatment or not. The first group was the spontaneous resolution group. The second group was the progression-surgery group. The mean hematoma volume in the spontaneous resolution group was $43.1 \mathrm{~mL}$. The mean degree of midline shift in the spontaneous resolution group was $5.3 \mathrm{~mm}$. The mean hematoma volume in the progression-surgery group was $62.0 \mathrm{~mL}$. The mean degree of midline shift in the second group was $6 \mathrm{~mm}$.

Conclusion : We suggest that the treatment modality should be determined according to the patient's symptoms and clinical condition and close observation could be performed in patients who do not have any symptoms or in patients who have mild to moderate headache without neurological deterioration.
\end{abstract}

Key Words : Close observation · Chronic subdural hematoma · Treatment.

\section{INTRODUCTION}

Chronic subdural hematoma (cSDH) is defined as a condition consisting of a slowly progressive accumulation of liquefied blood within the subdural space ${ }^{9)}$. This accumulation of blood in the subdural space may produce brain parenchymal compression, and at that time, patients have neurological symptoms such as headache, hypesthesia and hemiparesis. The incidence of cSDH increases significantly with age. The other risk factors of this disease have been established in many studies like taking blood thinners, long-term alcohol abuse, and history of head trauma ${ }^{23)}$.

$\mathrm{cSDH}$ is common and more prevalent in the aged population $^{9)}$. Surgical treatment might generally be indicated in conditions of large hematoma width $(>10 \mathrm{~mm}$ ) or shift of the midline (>5 mm) on brain computed tomography (CT) scan and surgical intervention has been accepted as the treatment of choice ${ }^{8)}$.
However, its outcomes may not be satisfactory because of recurrence and physical infirmity associated with aging ${ }^{26)}$. The reported rate of recurrence after surgery for $\mathrm{CSDH}$ is approximately $5-30 \%{ }^{11)}$.

When brain CT scan reveals slight compression of the brain parenchyma caused by hematoma and patients have no neurological symptoms or have mild symptoms, it is occasionally difficult to select the treatment strategy between surgical and conservative treatments and we cannot be sure about the surgical decision. Although many neurosurgeons are aware of spontaneously resolving subdural hematoma (SDH), the clinical and radiological characteristics have not yet been adequately described in the literature ${ }^{9)}$.

We reported 16 cases of close observation of cSDH. We compared and evaluated the clinical and radiological characteristics between the spontaneously cured cases and the cases requiring operation.

- Received : March 29, 2016 • Revised : July 25, 2016 •Accepted : August 12, 2016

- Address for reprints : Jung Ho Ko, M.D., Ph.D.

Department of Neurological Surgery, College of Medicine, Dankook University, 201 Manghyang-ro, Dongnam-gu, Cheonan 31116, Korea

Tel : +82-41-550-6280, Fax : +82-41-550-6034, E-mail : dkns0212@naver.com

- This is an Open Access article distributed under the terms of the Creative Commons Attribution Non-Commercial License (http://creativecommons.org/licenses/by-nc/3.0) which permits unrestricted non-commercial use, distribution, and reproduction in any medium, provided the original work is properly cited. 


\section{MATERIALS AND METHODS}

Clinical and radiological records of 241 patients who had presented with a low density to iso-dense $\mathrm{SDH}$ with parenchymal compression on brain CT scan between July 2005 and December 2014 were retrospectively reviewed. Patients were excluded if they had initially received surgery for $\mathrm{cSDH}$ or if they had presented with a low Glasgow coma scale (GCS) score below 8 due to difficulty in assessing the neurological status. From among these patients, we enrolled 16 patients who had received conservative treatment for $\mathrm{cSDH}$ without special medications which could affect hematoma resolution such as mannitol, steroids, tranexamic acid and angiotensin converting enzyme (ACE) inhibitors. Their brain CT scans showed the hematoma exerted a mass effect on the brain which may require surgical treatment such as burr-hole trephination with or without drainage. They were treated conservatively due to mild symptoms, their medical comorbidities, or their denial for operation. All patients had cSDH which was identified on the initial brain CT or some patients had a chronic stage of traumatic SDH which had progressed from the initially identified acute SDH.

We decided the treatment strategy for $\mathrm{CSDH}$ such as surgery or observation according to the clinical and radiological findings. If patients had neurological symptoms such as decreased mentality, motor weakness, sensory change or progressive severe headache, we preferred to perform surgical treatment. Depending on the patient's condition, we closely observed the patients and assessed the neurological status daily in case of admitted patients or performed the follow-up assessment once a week in outpatients. We preferred close observation in cases with high possibility of recurrence after the initial operation such as those with antiplatelet use and hematological disorder and in cases belonging to the high risk group of general anesthesia. We wanted to reduce the number of unnecessary operations due to operative or anesthetic complications. Therefore, we recommended nonsurgical treatment for the enrolled patients. We routinely performed follow-up brain CT once a week for 3 weeks and then once or twice a month until detection of nearly total resolution. Within the follow-up period, if neurologic symptoms had newly developed or progressed, brain CT was performed to consider surgery as soon as possible. Surgical methods were burr-hole trephination with drainage and saline irrigation. Generally, when it is decided to treat patients with $\mathrm{cSDH}$ surgically, the operation is carried out under local anesthesia. However, at our institution, considering the safety of patients and the possibility of lack of patients' cooperation during the operation, all surgeries were carried out under general anesthesia. Thus, prior to the treatment, it was inevitable to collect data regarding patient's comorbidities. During the observation period, we considered continuation of observation at every follow-up visit by comparing the change in hematoma amount and symptoms. We considered that the major criterion for surgical decision was the change in symptoms. Complete cure was defined when the SDH had nearly dis- appeared on follow-up brain CT.

The demographic data were collected after obtaining information on clinical factors; age, sex, history of trauma, past medical history such as diabetic mellitus, hypertension and medication especially antithrombotic agents, GCS, clinical symptoms and symptom duration, hematoma thickness and volume, degree of midline shift, accompanying intracranial lesion, Glasgow outcome scale (GOS) score. The patients received a CT scan of the brain at admission. We measured the volume of hematoma from the CT scans; all of these measurements were performed using the PACS imaging display software (Viewrex, TechHeim, Seoul, Korea). The volume measurement was hand-traced by the radiologist (D.S. Yoo) on each axial image, and then these areas were added together and multiplied by the slice thickness. The midline shift was identified as deviation of the septum pellucidum from the central position. The patients were classified according to the Markwalder's Grading Scale (MGS) ${ }^{18)}$. The grading score was used for patient classification and it ranged from 0 to IV. Grade 0 indicates neurologically intact patients. Grade I indicates alert and oriented patients who often complains of a headache. Grade II indicates drowsy or disoriented patients. Sometimes, the patients who are classified as having grade II have hemiparesis. Grade III indicates patients who are stuporous but respond appropriately to noxious stimuli and have hemiplegic symptom. The last grade, grade IV indicates patients who are comatose with absent motor responses to painful stimuli. They might have decerebrate or decorticate posturing.

The measured data were analyzed by using the $\mathrm{R}$ ver 3.2.4 software for Windows. We compared data such as the measured hematoma volume and the degree of midline shift. This comparison was performed by using the Wilcoxon rank sum test. The results were considered statistically significant if $p$ was $<0.05$.

\section{RESULTS}

Among these 16 patients, $13(81.3 \%)$ patients showed spontaneously resolved cSDH and 3 (18.7\%) patients received surgery due to symptom aggravation and growing hematoma. They were categorized into two groups based on whether they were cured with conservative treatment or not. The first group was the spontaneous resolution group (Table 1). There were 9 men and 4 women with a mean age of 62 years (range, 25 to 81 years). There were 8 patients over 65 years of age $(61.5 \%)$ and there were 5 below 65 years of age (38.5\%). Eight patients (61.5\%) had a medical history of hypertension, diabetes mellitus, hematologic disorder due to anaplastic anemia and alcohol abuse, anticoagulant use because of previous cerebral infarction or mitral valve replacement surgery and hemodialysis due to endstage renal disease. Ten patients $(76.9 \%)$ had a trauma history before admission. The most common presenting symptom was mild headache without neurological deficit. Case 2 patient only had hemiparesis. Their MGS ranged from grade I to grade II. The number of MGS grade I patients was 10 (76.9\%) and the 


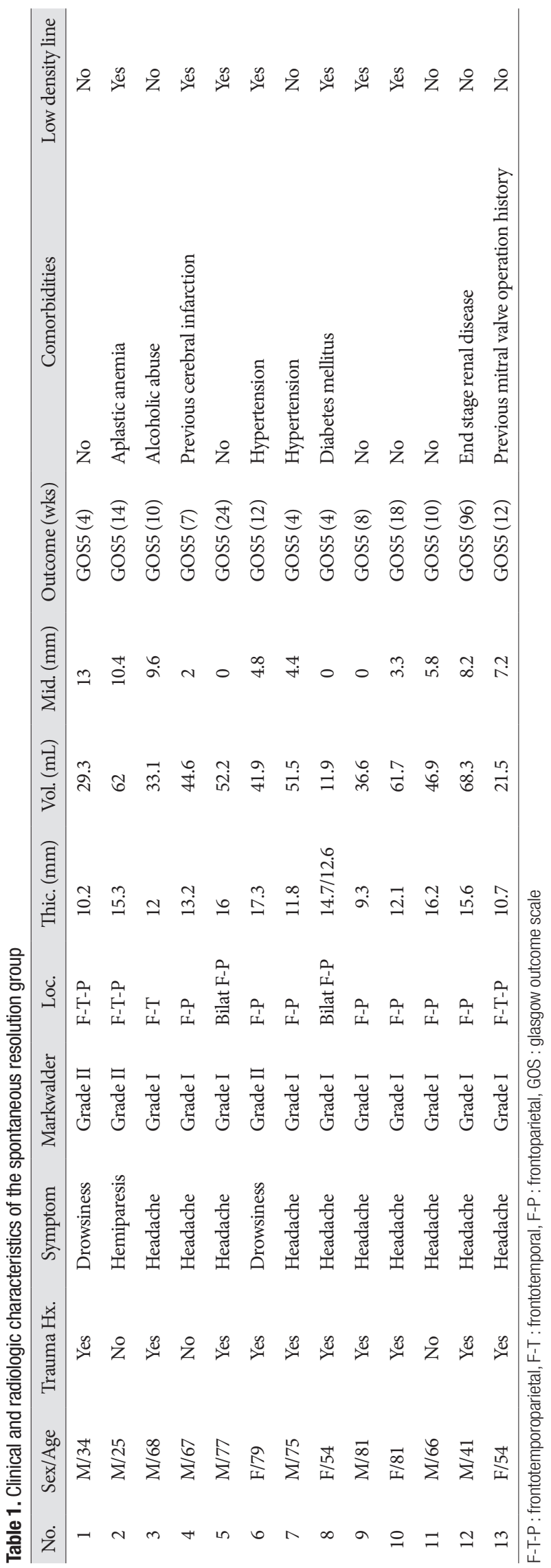

number of MGS grade II patients was 3 (23.1\%). The fronto-parietal area was the most common area of hematoma involvement. The mean hematoma volume was $43.1 \mathrm{~mL}$ (range, 11.9 to $68.3 \mathrm{~mL}$ ). The mean hematoma thickness on brain CT was $13.3 \mathrm{~mm}$ (range, 9.3 to $17.3 \mathrm{~mm}$ ). The mean degree of midline shift was $5.3 \mathrm{~mm}$ (range, 0 to $10.4 \mathrm{~mm}$ ). All patients had good outcomes (GOS 5) and their clinical symptoms improved. There were no recurrences of $\mathrm{cSDH}$. Mean duration from identification to complete resolution of cSDH was 17 weeks (range, 4 to 96 weeks). The second group was the progression-surgery patients. Table 2 summarizes their demographic data. There were 2 men and 1 woman with a mean age of 72 years (range, 67 to 81 years). One patient had a history of antiplatelet medication. All patients had a trauma history before admission. All patients presented with mild headache without neurological deficit. The mean hematoma volume was $62.0 \mathrm{~mL}$ (range, 41.0 to $75.0 \mathrm{~mL}$ ). The mean hematoma thickness was $17.5 \mathrm{~mm}$ (range, 14.3 to $21.5 \mathrm{~mm}$ ). The mean degree of midline shift was 6.0 $\mathrm{mm}$ (range, 0 to $15.0 \mathrm{~mm}$ ). Mean duration from the decision of surgery for hematoma was 5.5 weeks (range, 2 to 8 weeks). All patients had good outcomes (GOS 5) and their clinical symptoms improved. There were no recurrences of $\mathrm{CSDH}$.

The results of univariate analysis with the Wilcoxon rank sum test are described in Table 3. The hematoma volumes showed no significant difference between the two groups. However, the point estimates of the median in the spontaneous resolution group and the progression-surgery group were 44.6 and 70 , respectively. The $p$-value for this analysis was 0.146 . This study is limited in its significance due to the small sample size. Therefore, further analysis with a larger sample size for strengthening statistical power is needed to strengthen our results ${ }^{15)}$.

\section{Case illustration}

\section{A 75-year-old male}

The patient was admitted due to minor head trauma. He complained of mild headache and had an alert mentality. The initial brain CT revealed a scanty acute SDH in the right hemisphere (Fig. 1A). He was treated conservatively and his clinical course was uneventful. Follow-up brain CT performed on the 20th hospital day (HD) showed a liquefied SDH that compressed the brain parenchyma with a mild midline shift (Fig. 1B). The measured volume of the hematoma was $51.5 \mathrm{~mL}$ and the length of midline shift was $4.4 \mathrm{~mm}$. Because his symptom had been improving slowly and he did not have any other neurological signs, we made a decision of performing close observation. Follow-up brain CT scan performed on the 46th HD showed an improved mass effect and a remarkably decreased amount of hematoma (Fig. 1C). He was discharged at that time and brain CT scan was acquired in the outpatient department 2 weeks later. The final brain CT scan revealed nearly complete resolution of the hematoma (Fig. 1D). He has not experienced any recurrence of symptom and hematoma. 


\section{A 25-year-old male}

The patient had aplastic anemia which was diagnosed 10 years ago and he was frequently treated by blood transfusion due to a hematological disorder. He presented with left side hemiparesis and headache. The initial brain CT scan showed a mixed subacute and cSDH that compressed the brain parenchyma and caused a midline shift (Fig. 2A). The measured volume of the hematoma was $62.0 \mathrm{~mL}$ and the length of midline shift was $10.4 \mathrm{~mm}$. He had severe thrombocytopenia (measured platelet count was $31000 / \mathrm{uL}$ ) which might have led to spontaneous bleeding and his medical condition was very poor. Be- cause we were concerned about hemostasis and poor general condition, we recommended conservative treatment and informed consent was obtained from the patient and his relatives. Close observation and conservative treatment including transfusion were performed in the intensive care unit. His neurological status was not aggravated and the clinical course was uneventful for 2 weeks. The hemiparesis recovered slowly from the 8th HD onwards. Follow-up brain CT performed on the 14th HD revealed decreased amount of hematoma and mass effect (Fig. 2B). His neurologic symptom disappeared on the 30th $\mathrm{HD}$, and brain CT scan revealed a remarkable reduction in he-

Table 2. Clinical and radiologic characteristics of the progression-surgery group

\begin{tabular}{cccccccccccc}
\hline No. & Sex/Age & Trauma Hx. & Symptom & Markwalder & Loc. & Thic. $(\mathrm{mm})$ & Vol. $(\mathrm{mL})$ & Mid. $(\mathrm{mm})$ & $\begin{array}{c}\text { Outcome } \\
\text { (wks) }\end{array}$ & Comorbid & $\begin{array}{c}\text { Low } \\
\text { density line }\end{array}$ \\
\hline 1 & M/81 & Yes & Headache & Grade I & Bilat F-T-P & $21.5 / 18.2$ & $44 / 70$ & 0 & OP & Hypertension & Yes \\
2 & F/67 & Yes & Headache & Grade I & F-T-P & 14.3 & 41 & 15 & OP & Hypertension & No \\
3 & M/69 & Yes & Dizziness & Grade I & F-P & 16.2 & 75 & 3 & OP & No & No \\
\hline
\end{tabular}

OP : operation, F-T-P : frontotemporoparietal, F-P : frontoparietal

Table 3. The univariate analysis of the hematoma volume between two groups

\begin{tabular}{|c|c|c|c|c|c|c|c|}
\hline & \multicolumn{3}{|c|}{ Spontaneously resolution group $(\mathrm{n}=13)$} & \multicolumn{3}{|c|}{ Progression-surgery group $(\mathrm{n}=3)$} & \multirow{2}{*}{$p$-value } \\
\hline & Mean & SD & Median & Mean & SD & Median & \\
\hline Hematoma volume $(\mathrm{mL})$ & 43.2 & 16.5 & 44.6 & 62 & 18.4 & 70 & 0.146 \\
\hline
\end{tabular}

${ }^{*} p$-value was calculated by using Wilcoxon rank sum test. SD : standard deviation
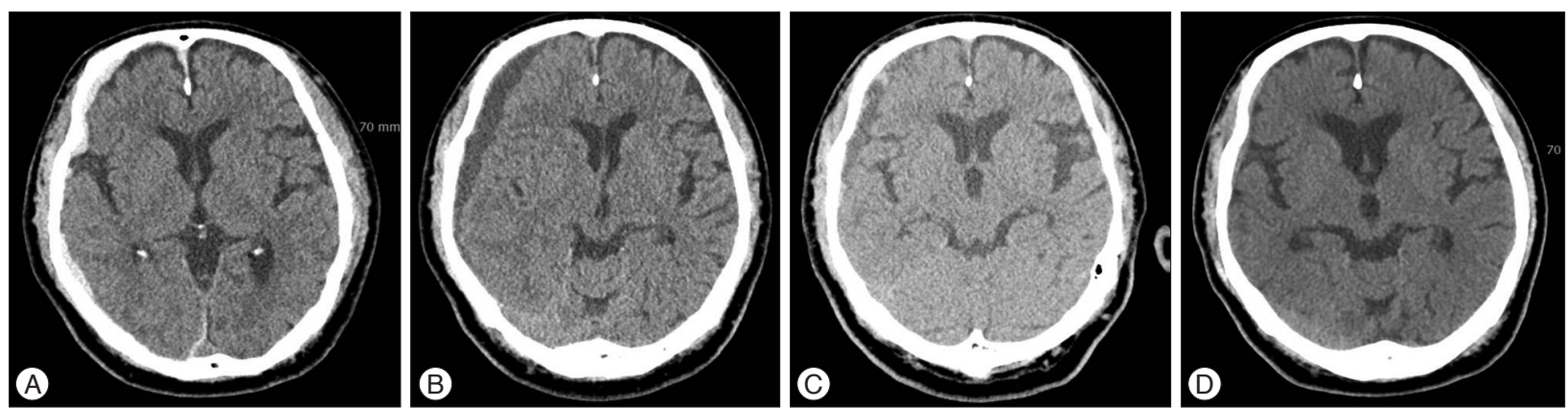

Fig. 1. A 75-year-old male complained of mild headache and he had alert mentality after minor head trauma [Table 1 (Case 7)]. A : Initial brain CT reveals scanty acute SDH in the right hemisphere. B : Follow-up brain CT scan on the 20th hospital day (HD) shows liquefied subdural hematoma that compressed the brain parenchyma with a mild midline shift. C : Follow-up brain CT scan on the 46th HD shows an improved mass effect and a remarkably decreased amount of hematoma. D : Final brain CT scan reveals nearly complete resolution of the hematoma. SDH : subdural hematoma.
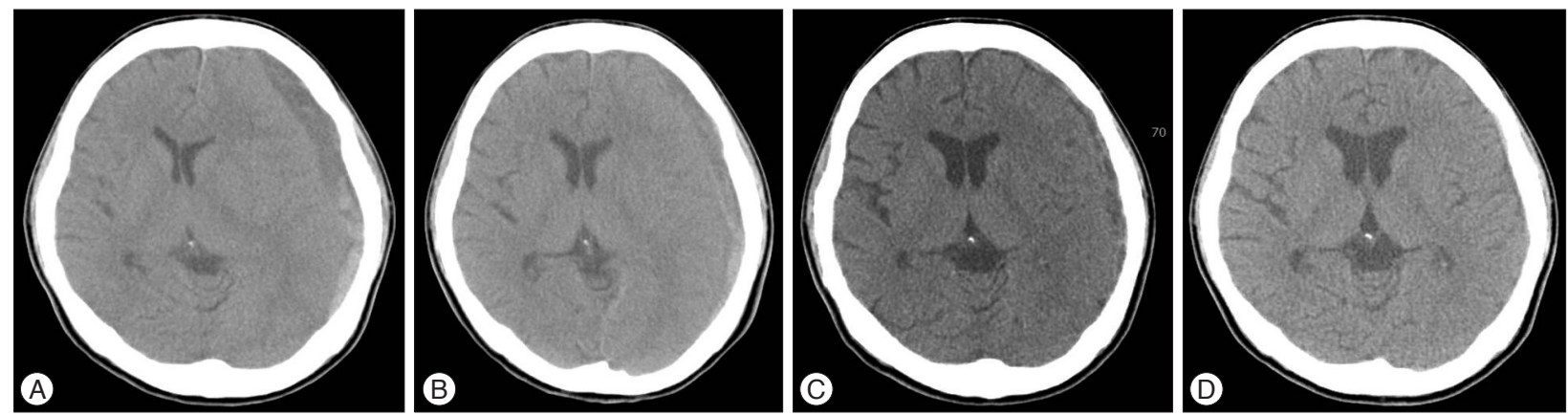

Fig. 2. A 25-year-old male presented with left side hemiparesis and headache [Table 1 (Case 2)]. A : Initial brain CT scan shows that a mixed subacute to chronic subdural hematoma compresses the brain parenchyma and causes a midline shift. B : Follow-up brain CT scan on the 14th hospital day $(\mathrm{HD})$ reveals decreased amount of hematoma and mass effect. C : Follow-up brain CT scan on the 30th HD identifies remarkable reduction in hematoma volume. D : Final brain CT scan shows complete disappearance of the hematoma. 
matoma volume (Fig. 2C). After discharge, brain CT scan was acquired in the outpatient department 1 month later. The final brain CT scan showed that the hematoma had completely disappeared (Fig. 2D).

\section{An 81-year-old male}

He was brought to our institution via the neurosurgical outpatient department. The brain CT scan revealed an iso-dense lesion in the subdural space in the bilateral convexity (Fig. 3A). He presented with unspecific myalgia without neurological symptoms and signs. We explained to his relative that this lesion had a chance of growing into a hematoma. Close observation was performed. During the admission period, he did not have aggravation of symptoms. After 2 weeks, follow-up CT scan showed no change compared with the previous CT scan. We decided to discharge him and perform outpatient follow-up. At the first follow-up after discharge, his relative informed that the patient did not prefer to talk with other people as found in people with mutistic nature. Brain CT scan showed an increased amount of hematoma (Fig. 3B). Surgical evacuation of the hematoma was performed and the patient fully recovered (Fig. 3C).

\section{DISCUSSION}

\section{The pathophysiologic mechanism of hematoma growth}

The cSDH tends to gradually increase in volume. Several hypotheses about the mechanism of increase in the hematoma volume have been proposed since a long time. One hypothesis is the osmotic pressure theory after formation of internal and external capsules. As the hematoma volume increases, tension in the hematoma capsule is increased followed by micro-tear of its capillary vessels. Then hemorrhage occurs and it becomes a factor involved in increasing the osmotic pressure in the hematoma $^{25)}$. This osmotic theory recently been doubted due to the following factors : 1) there was no significant increase in the volume in this theory when hematoma membranes were used;
2) fresh erythrocytes were always introduced in the hematoma fluid on repeated tapping; 3 ) it was not possible to prove that the arachnoid acts as a membrane permeable to cerebrospinal fluid; and 4) it has been shown that albumin, the most osmotically active protein, cannot be found in destroyed red blood cells but is derived from the plasma ${ }^{17)}$. This previously accepted hypothesis has been proven wrong ${ }^{4,28)}$. Rather, hematoma expansion is thought to result from repeated micro-hemorrhage from the fragile neo-membranes ${ }^{4,13)}$. The phenomenon of recurrent bleeding from the hematoma capsule as an etiological factor for the development of chronic SDH was assumed by Putnam and Cushing in 1932, and later by Dandy. This theory was corroborated by the experimental studies performed by Markwalder. ${ }^{17)}$. Currently, various clinical and experimental studies indicate that fibrinolysis and liquefaction of the initial clot occur rapidly and subsequently inhibit the blood coagulation system in the hematoma. Additionally, the byproducts of blood breakdown and the fibrinolytic process also cause thickening of the inner dural layer with ingrowing neocapillaries which play an important role in the leakage of blood, causing enlargement of the subdural collection due to microhemorrhage and further increase in the fibrinolytic activity. The correlation between this cycle of re-bleeding and fibrinolysis, and reabsorption of the subdural collection will determine whether the $\mathrm{CSDH}$ will resolve, persist or enlarge ${ }^{24)}$. Some authors stated that the hyperfibrinolytic activity has been proved to be critical for liquefaction of the hematoma and progression of $\mathrm{cSDH}$. Several studies have indicated the hyperfibrinolytic and coagulative activity in $\mathrm{CSDH}$ and some studies have shown that increased permeability of the capillaries in the hematoma outer membrane can influence the enlargement of a $\mathrm{cSDH}^{5)}$. Based on this rationale, they applied an antifibrinolytic agent for prevention of enlargment and recurrence of $\mathrm{cSDH}^{11)}$.

\section{Close observation study}

Previous reports on spontaneous resolution of cSDH without
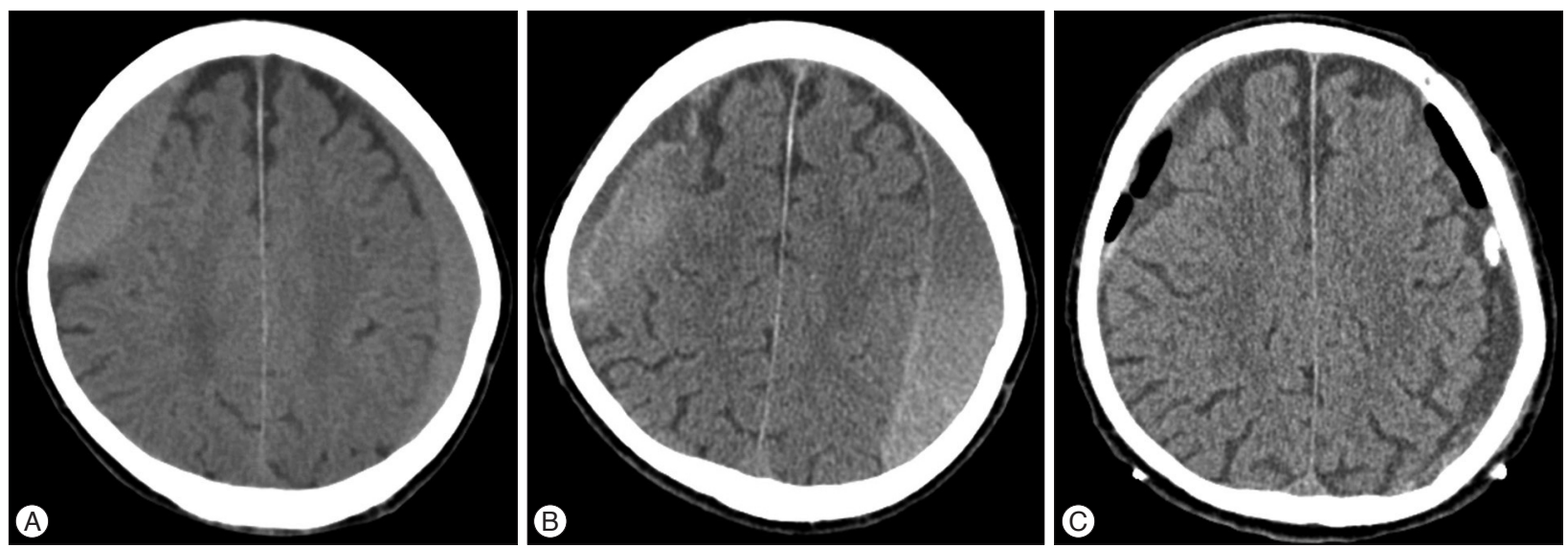

Fig. 3. An 81-year-old male presented with an incidental subdural hematoma Table 2 (Case 1)]. A : Initial brain CT scan reveals an isodense lesion in the subdural space in bilateral convexity. B : When the symptom changed, follow-up brain CT shows an increased amount of hematoma. C : Postoperative brain CT scan shows reduction of hematoma through drainage catheters. 
Table 4. Previous reports about spontaneous resolution of chronic subdural hematoma without special medication or surgery

\begin{tabular}{|c|c|c|c|c|c|c|c|c|c|c|}
\hline No. & Author/Year & Sex/Age & $\begin{array}{c}\text { Trauma } \\
\mathrm{Hx} .\end{array}$ & Symptom & Markwalder & Loc. & $\begin{array}{l}\text { Thic. } \\
(\mathrm{mm})\end{array}$ & $\begin{array}{l}\text { Vol. } \\
(\mathrm{mL})\end{array}$ & $\begin{array}{l}\text { Mid. shift } \\
(\mathrm{mm})\end{array}$ & $\begin{array}{l}\text { Outcome } \\
\text { (wks) }\end{array}$ \\
\hline 1 & Naganuma et al. ${ }^{20)} / 1986$ & $79 / \mathrm{M}$ & Yes & No & Grade 0 & $\mathrm{~F}$ & 20 & - & - & Improved (33) \\
\hline 2 & Naganuma et al. & $75 / \mathrm{M}$ & Yes & GCS14 & Grade I & $\mathrm{F}$ & 16 & - & - & Improved (46) \\
\hline 3 & Naganuma et al. & $41 / \mathrm{M}$ & Yes & Somnolence & Grade II & $\mathrm{F}-\mathrm{P} / \mathrm{O}$ & $34 / 64$ & - & - & Improved (35) \\
\hline 4 & Naganuma et al. & $21 / \mathrm{M}$ & Yes & No & Grade 0 & $\mathrm{~F}$ & 9 & - & - & Improved (11) \\
\hline 5 & Horikoshi et al. ${ }^{9)} / 1998$ & $\mathrm{~F} / 75$ & Yes & Double vision & Grade I & $\mathrm{F}$ & 12 & - & 5 & Improved (20) \\
\hline 6 & Horikoshi et al. & $\mathrm{M} / 69$ & Yes & Dizziness & Grade I & Bil. F & $13 / 18$ & - & 0 & Improved (11) \\
\hline 7 & Horikoshi et al. & $\mathrm{M} / 68$ & - & Headache & Grade I & $\mathrm{F}$ & 10 & - & 5 & Improved (-) \\
\hline 8 & Horikoshi et al. & $\mathrm{F} / 75$ & - & Headache & Grade I & $\mathrm{F}$ & 15 & - & 0 & Improved (-) \\
\hline 9 & Parlato et al. ${ }^{22} / 2000$ & $\mathrm{M} / 79$ & Yes & Unstable gait & Grade II & F-P & - & - & 0 & Improved (7) \\
\hline 10 & Parlato et al. & $\mathrm{M} / 68$ & Yes & Headache & Grade I & F-T-P & - & - & 0 & Improved (6) \\
\hline 11 & Parlato et al. & $\mathrm{F} / 76$ & Yes & Headache & Grade I & F-P & - & - & 0 & Improved (6) \\
\hline 12 & Parlato et al. & $\mathrm{M} / 72$ & Yes & Headache & Grade I & F-P & - & - & 0 & Improved (6) \\
\hline 13 & Parlato et al. & $\mathrm{F} / 71$ & Yes & Headache & Grade I & F-P & - & - & 0 & Improved (6) \\
\hline 14 & Göksu et al. $.^{7} / 2009$ & $\mathrm{M} / 35$ & Yes & Speech disturbance & Grade II & F-P & - & 76.5 & 0 & Improved (19) \\
\hline 15 & Marcikić et al. ${ }^{16)} / 2010$ & $\mathrm{~F} / 76$ & Yes & Unstable gait & Grade II & F-P & - & - & - & Improved (1) \\
\hline 16 & Juković et al. ${ }^{10)} / 2012$ & $\mathrm{~F} / 65$ & Yes & Disorientation & Grade II & F-P & 17 & - & 7 & Improved (28) \\
\hline 17 & Baldawa et al. ${ }^{1)} / 2015$ & $\mathrm{M} / 65$ & - & Hemiparesis & Grade II & F-P & 15 & - & 8 & Improved (96) \\
\hline 18 & Lee et al. ${ }^{12)} / 2015$ & $\mathrm{~F} / 87$ & Yes & Hemiparesis & Grade II & F-T-P & 22 & - & 12 & Improved (20) \\
\hline
\end{tabular}

F : frontal, 0 : occipital, F-T-P : frontotemporoparietal, F-P : frontoparietal, GCS : glasgow coma scale

special medication and surgery have been published ${ }^{1,7,9,10,12,16,20,22)}$. Most of them were case studies which were composed of small cases series with 4 or 5 cases in one report. To the best of our knowledge, our study has the highest number of enrolled cases compared to the studies reported so far, and unlike other investigations, this study has a unique aspect as it considered the rate of spontaneous resolution of cSDH. Table 4 presents the list and characteristics of previous studies. The total number of all collected cases was eighteen. The mean age of this group was 66.5 years (range, 21 to 87 years). There were 11 men (61.1\%) and 7 women $(38.9 \%)$. Most of them, except for 3 patients had a trauma history such as traffic accident, fall, and blunt trauma. All patients had symptoms such as headache, dizziness, drowsiness and unstable gait. Two patients had a mild hemiparesis and one patient had diplopia. The most commons site was the frontal area. Mean hematoma thickness was $16.7 \mathrm{~mm}$ (range, 9 to 34 $\mathrm{mm}$ ) and there was no data for thickness in 7 cases. The average degree of midline shift was $2.8 \mathrm{~mm}$ (range, 5 to $12 \mathrm{~mm}$ ). All of the patients had a good clinical course. Based on this table, their results were similar to our results.

Naganuma et al. ${ }^{20)}$ reported 4 cases of spontaneous resolution of cSDHs and suggested the three following characteristic CT findings associated with spontaneous resolution of chronic $\mathrm{SDH}$ : low density hematoma or iso-dense hematoma, small size, and ventricular dilatation. They considered that the dilated ventricle could cause elevated counterpressure against cSDH. This theory might have some role in the resolution of SDH. We reviewed the radiological data of the spontaneous resolution group. Except for one case, there were no other cases of ventric- ular dilatation in this group and the mean Evan's ratio ranged from 0.19 to 0.32 .

Horikoshi et al. ${ }^{9)}$ reported that the clinical and radiological findings of four cases (five hematomas) were compared to those of 19 surgical cases. They considered that spontaneously resolving cSDH were asymptomatic or only caused mild transient headache, and disappeared within 4 to 9 months after head injury. All spontaneously resolving SDHs were located in the frontal region, and maximum thickness and midline displacement were less $5 \mathrm{~mm}$. Our results were similar to their results. Table 1 shows that the most common location of $\mathrm{cSDH}$ was the frontal region. The mean value of midline displacement in our study was 5.2 $\mathrm{mm}$ and thickness was $13.3 \mathrm{~mm}$. We suggest that close observation could be chosen when hematoma volume or thickness is below $43 \mathrm{~mL}$ and $13 \mathrm{~mm}$, respectively, and midline shift is below $5 \mathrm{~mm}$ on brain CT scans. According to our results, there were no statistically significant differences between the two groups. However, the spontaneous resolution group had a relatively less amount of hematoma. This shows that the patients who have a definite large amount of $\mathrm{cSDH}(>43 \mathrm{~mL})$ should be informed about the possibility of surgery during the close observation period. Especially, Horikoshi et al. ${ }^{11)}$ suggested that brain CT scan demonstrated a low density line between the hematoma and the cerebral cortex, indicative of remaining cerebrospinal fluid space in four of five hematomas ${ }^{9}$. In our study, in 7 patients (53.8\%) of the spontaneously resolution group, the low density line on the initial brain CT scans was visible. While in only one patient (33.3\%) of the progression-surgery group, the low density line was visible. 
Some authors suggested that age over 70 years, worsening mental function, the presence of brain atrophy, and absence of clinical and radiological symptoms due to increased intracranial pressure are clinical and radiological findings that allow one to choose conservative therapy. In our study, the number of patients who were aged over 70 years was 5 (38.5\%) in the spontaneously resolution group. However, in this aging society, age cannot be an independent factor that affects the decision making process. Many physicians should be cautious about patients' symptoms. If the symptoms have not begun to resolve after 7 or 10 days of clinical observation, surgery should be performed immediately in patients with progressive neurological deficits and increase in size of the SDH regardless of the age $\mathrm{e}^{22}$. We suggest that the treatment modality could be determined according to the patient's symptoms, and the clinical indication for close observation is patients who do not have any symptoms or patients who have moderate headache without neurological deterioration (MGS 0 or I).

Nakamura et al. ${ }^{21)}$ stated that the decreased fibrinolytic activity of the hematoma capsule and the fluid might have caused a spontaneous resolution. They also noted that the resolving hematoma appeared as a low density area or an area of decreasing density, from mixed to low, in successive CT scans.

\section{Spontaneous resolution rate of $\mathrm{CSDH}$}

Miranda et al. ${ }^{19)}$ reported about the elderly patients who suffered from cSDH. Of the 209 cases, primary surgical intervention was performed in 137 (65.5\%) patients. The remaining 72 (34.5\%) patients were simply observed. In this report, the natural course or results obtained in the simply observed patients were not described in detail. Lee et al. ${ }^{14)}$ reported about the forty patients who were admitted to their institution due to $\mathrm{CSDH}$ and finally only two (5\%) patients had a spontaneously absorbed cSDH. These results were comparable to our results. Finally, the spontaneous resolution rate was $5.3 \%(13 / 241)$ in our study.

\section{The kind of conservative treatment with special medication and its rationale}

Some authors believed that only reduction in the internal pressure of a hematoma will cure $\mathrm{cSDH}$, and therefore, the problem may be solved nonsurgically if the internal pressure within a SDH can be reduced by any method, not necessarily craniotomy. Hence, nonsurgical treatment consisting of osmotherapy with $20 \%$ mannitol was performed in a consecutive series. Disappearance or marked reduction of the hematoma content and complete clinical recovery were obtained in 22 out of 23 patients ${ }^{25)}$. Sun et al. ${ }^{24)}$ reported regarding corticosteroid treatment for $26 \mathrm{cSDH}$ patients who had GCS score of 15 with no neurological deficits, but they had symptoms such as headache or unsteady gait (MGS I). Among them, only one case (4\%) required surgical drainage. They supported the rationale for dexamethasone treatment suggested by experimental studies that the development of cSDH is due to an inflammatory process induced by the presence of erythrocyte breakdown products in the subdural space ${ }^{24)}$, and they showed that corticosteroids inhibited the formation of protein permeable membranes and decreased the size of chronic hematomas in rats ${ }^{6}$. Also, Delagado-López et $\mathrm{al}^{3)}$ reported that although $21.8 \%$ of the patients on dexamethasone ultimately required surgical drain, a favorable outcome was obtained in $96 \%$ of the patients who had MGS I and II. The rationale for the use of dexamethasone in $\mathrm{CSDH}$ lies in its antiangiogenic properties over the subdural clot membrane, as it is derived from experimental studies and very few clinical observations have been published. Surgical evacuation of $\mathrm{cSDH}$ is known to achieve excellent results, but there are no well-designed trials comparing medical versus surgical therapies ${ }^{3)}$. In the study by Delgado-López et al. ${ }^{3)}$, they suggested that the only indication for surgery as an initial emergency treatment may be the case of a patient with depressed level of consciousness and severe neurological deterioration occurring acutely and it seems reasonable to propose the steroid trial as the first therapeutic choice. Even though they conservatively treated MGS II patients using corticosteroids, their outcome was almost favorable. We think it may be a noteworthy result, and when patient has a moderate neurologic deficit or decreased mentality and if the patient's condition is very poor to undergo surgery, corticosteroid treatment could be chosen. In a recent report, tranexamic acid has been shown to prevent the early stages of cSDH that can occur after head trauma and the recurrence of cSDH after surgery. Tranexamic acid is an antifibrinolytic agent that has fewer side effects than other agents and is widely used for hemostasis. In several studies assessing the role of hyperfibrinolytic activities in the liquefaction and enlargement of $\mathrm{cSDH}^{5)}$, they hypothesized that tranexamic acid would inhibit the hyperfibrinolytic activity within the $\mathrm{cSDH}^{11)}$. Aberrant angiogenesis and localized inflammation contribute to the formation of $\mathrm{cSDH}$. Atorvastatin is active in promoting angiogenesis and modulating inflammation ${ }^{26)}$. There is increasing evidence that impaired angiogenesis in the neomembrane and localized inflammation play a key role in the formation of a cSDH. Impaired angiogenesis results in blood leakage from immature vessels of the neomembrane and localized inflammation hampers angiogenesis and prevents leaked blood from being absorbed. 3-Hydroxy3-methylglutaryl-Coenzyme A reductase inhibitors, which are the first-line treatment in patients with high cholesterol and coronary heart disease, have been demonstrated to improve angiogenesis and reduce inflammation ${ }^{26)}$. The researchers reported that 22 of the 23 patients experienced improvements in symptoms, and reduction in hematoma volume within the first month of the treatment. Hematoma was completely resolved in 17 patients (77.3\%) and shrank in 5 patients (22.7\%) within 3 months after the treatment was initiated ${ }^{26)}$. Because the $\mathrm{CSDH}$ is characterized by pathological vascularization of the parietal membrane, plasma leakage from immature vessels may be involved in hematoma enlargement and recurrence. The researchers tested the hypothesis that the antiangiogenic mechanism of ACE- 
inhibitor treatment for the control of arterial hypertension reduces the risk of recurrence of $\mathrm{cSDH}$. Their data suggest that ACE-inhibitor treatment for the control of arterial hypertension lowers the risk of recurrence in patients undergoing operation for $\mathrm{CSDH}$ and possibly even the development of cSDH. This effect might be the result of an antiangiogenic mechanism of ACEinhibitors ${ }^{27}$.

\section{Drawbacks of nonsurgical treatment}

In comparison with surgical treatment, the nonsurgical treatment has several disadvantages. First, the prolonged presence of a hematoma on the cerebral surface even with low pressure may raise fears of an ill effect on cerebral function. The usual surgical treatment eliminates the hematoma as soon as it is discovered, while nonsurgical treatment cannot eliminate a mass lesion by one effort ${ }^{25}$. The second drawback is the side effect of each medication. The third drawback is that longer follow-up periods may be required. Because $\mathrm{CSDH}$ frequently occurs in the elderly group, special attention should be paid to this side effect. The mannitol treatment is not completely free from problems. It should be performed with combined sufficient hydration and with careful attention being paid to the electrolyte balance. The side effect of long-term use of corticosteroids had been widely accepted. Gastric mucosal hemorrhage, edema, and increased risk of infection are associated with use of steroids ${ }^{2}$. Also, tranexamic acid had several side effects including gastrointestinal symptoms and ischemic events. ACE inhibitors, which comprise a class of anti-hypertensive medications, may have unsolicited influence on blood pressure, and their effect on $\mathrm{CSDH}$ is yet to be proven ${ }^{27}$.

\section{Limitations}

The present study has some limitations. First, the present study is performed by using a retrospective chart review. This method may increase the risk of selection bias. Second, the number of patients enrolled in this study is too small to reach a statistical significance. Further study with a large number of patients is needed to establish the criteria for conservative management. Despite these limitations, when physicians encounter a similar situation, our study might provide guidance in selecting the treatment modality.

\section{CONCLUSION}

We think that spontaneously resolving SDH might be more frequent than formerly expected. We suggest that the treatment modality should be determined according to the patient's symptoms and clinical condition and close observation could be performed in patients who do not have any symptoms or in patients who have mild to moderate headache without neurological deterioration (MGS grade 0 or I). When a patient has a moderate neurologic deficit or decreased mentality and if the patient's condition is too poor to undergo surgery, corticosteroid treatment could be chosen. Also, we suggest that close observation could be chosen when hematoma volume or thickness is below $43 \mathrm{~mL}$ or $13 \mathrm{~mm}$, respectively, and midline shift is below $5 \mathrm{~mm}$ on brain CT scan.

\section{References}

1. Baldawa SS, Nayak $\mathrm{N}$ : Spontaneous resolution of bilateral chronic subdural hematoma. Turk Neurosurg 25 : 835-836, 2015

2. Berghauser Pont LM, Dirven CM, Dippel DW, Verweij BH, Dammers $\mathrm{R}$ : The role of corticosteroids in the management of chronic subdural hematoma : a systematic review. Eur J Neurol 19 : 1397-1403, 2012

3. Delgado-López PD, Martín-Velasco V, Castilla-Díez JM, RodríguezSalazar A, Galacho-Harriero AM, Fernández-Arconada O : Dexamethasone treatment in chronic subdural haematoma. Neurocirugia (Astur) $20: 346-359,2009$

4. Ducruet AF, Grobelny BT, Zacharia BE, Hickman ZL, DeRosa PL, Andersen $\mathrm{KN}$, et al. : The surgical management of chronic subdural hematoma. Neurosurg Rev 35 : 155-169; discussion 169, 2012

5. Fujisawa H, Ito H, Kashiwagi S, Nomura S, Toyosawa M : Kallikrein-kinin system in chronic subdural haematomas : its roles in vascular permeability and regulation of fibrinolysis and coagulation. J Neurol Neurosurg Psychiatry 59: 388-394, 1995

6. Glover D, Labadie EL : Physiopathogenesis of subdural hematomas. Part 2 : inhibition of growth of experimental hematomas with dexamethasone. J Neurosurg 45 : 393-397, 1976

7. Göksu E, Akyüz M, Uçar T, Kazan S : Spontaneous resolution of a large chronic subdural hematoma : a case report and review of the literature. Ulus Travma Acil Cerrahi Derg 15 : 95-98, 2009

8. Greenberg MS : Handbook of Neurosurgery, ed 7. New York : Thieme, 2010, pp899-902

9. Horikoshi T, Naganuma H, Fukasawa I, Uchida M, Nukui H : Computed tomography characteristics suggestive of spontaneous resolution of chronic subdural hematoma. Neurol Med Chir (Tokyo) 38 : 527-532; discussion 532-533, 1998

10. Juković M, Kojadinović Z, Popovska B, Till V : Complete spontaneous resolution of compressive chronic subdural hematoma in a patient with liver failure. Med Glas (Zenica) 9: 417-420, 2012

11. Kageyama H, Toyooka T, Tsuzuki N, Oka K : Nonsurgical treatment of chronic subdural hematoma with tranexamic acid. J Neurosurg 119 : 332-337, 2013

12. Lee GS, Park YS, Min KS, Lee MS : Spontaneous resolution of a large chronic subdural hematoma which required surgical decompression. J Korean Neurosurg Soc 58 : 301-303, 2015

13. Lee JY, Ebel H, Ernestus RI, Klug N : Various surgical treatments of chronic subdural hematoma and outcome in 172 patients : is membranectomy necessary? Surg Neurol 61 : 523-527; discussion 527-528, 2004

14. Lee TS, Yang DD, Sung KW, Kim JO, Lee WH, Rhee HY : Two cases of spontaneously absorbed chronic subdural hematoma. J Korean Neurosurg Soc 15 : 861-866, 1986

15. Lieber RL : Statistical significance and statistical power in hypothesis testing. J Orthop Res 8 : 304-309, 1990

16. Marcikić M, Hreckovski B, Samardzić J, Martinović M, Rotim K : Spontaneous resolution of post-traumatic chronic subdural hematoma : case report. Acta Clin Croat $49: 331-334,2010$

17. Markwalder TM : Chronic subdural hematomas : a review. J Neurosurg 54 : 637-645, 1981

18. Markwalder TM, Steinsiepe KF, Rohner M, Reichenbach W, Markwalder $\mathrm{H}$ : The course of chronic subdural hematomas after burr-hole craniostomy and closed-system drainage. J Neurosurg 55 : 390-396, 1981

19. Miranda LB, Braxton E, Hobbs J, Quigley MR : Chronic subdural hematoma in the elderly : not a benign disease. J Neurosurg 114: 72-76, 2011 
20. Naganuma H, Fukamachi A, Kawakami M, Misumi S, Nakajima H, Wakao T: Spontaneous resolution of chronic subdural hematomas. Neurosurgery $19: 794-798,1986$

21. Nakamura N, Ogawa T, Hashimoto T, Yuki K, Kobayashi S : [Reevaluation on resolving subdural hematoma (author's transl)]. Neurol Med Chir (Tokyo) 21 : 491-500, 1981

22. Parlato C, Guarracino A, Moraci A : Spontaneous resolution of chronic subdural hematoma. Surg Neurol 53 : 312-315; discussion 315-317, 2000

23. Sim YW, Min KS, Lee MS, Kim YG, Kim DH : Recent changes in risk factors of chronic subdural hematoma. J Korean Neurosurg Soc 52 : 234-239, 2012

24. Sun TF, Boet R, Poon WS : Non-surgical primary treatment of chronic subdural haematoma : preliminary results of using dexamethasone. Br J
Neurosurg 19: 327-333, 2005

25. Suzuki J, Takaku A : Nonsurgical treatment of chronic subdural hematoma. J Neurosurg 33 : 548-553, 1970

26. Wang D, Li T, Tian Y, Wang S, Jin C, Wei H, et al. : Effects of atorvastatin on chronic subdural hematoma : a preliminary report from three medical centers. J Neurol Sci 336 : 237-242, 2014

27. Weigel R, Hohenstein A, Schlickum L, Weiss C, Schilling L : Angiotensin converting enzyme inhibition for arterial hypertension reduces the risk of recurrence in patients with chronic subdural hematoma possibly by an antiangiogenic mechanism. Neurosurgery $61: 788-792$; discussion 792-793, 2007

28. Weir B : The osmolality of subdural hematoma fluid. J Neurosurg 34 : 528-533, 1971 